\title{
UPLC-MS/MS and Network Pharmacology-Based Analysis of Bioactive Anti-Depression Compounds in Betel Nut
}

\author{
Yunjia Yang (D) ${ }^{1, *}$ \\ Hairong Huang ${ }^{1} * *$ \\ Zeying Cui ${ }^{2}$ \\ Jun $\mathrm{Chu}^{3}$ \\ Guankui Du (D) $2,4,5$
}

\begin{abstract}
'School of Public Health, Hainan Medical University, Haikou, People's Republic of China; ${ }^{2}$ Key Laboratory of Molecular Biology, Hainan Medical University, Haikou, People's Republic of China; ${ }^{3}$ Key Laboratory of Xin'an Medicine, Ministry of Education, Anhui University of Chinese Medicine, Hefei, Anhui, People's Republic of China; ${ }^{4}$ Department of Biochemistry and Molecular Biology, Hainan Medical University, Haikou, People's Republic of China; ${ }^{5}$ Biotechnology and Biochemisty Laboratory, Hainan Medical University, Haikou, People's Republic of China

*These authors contributed equally to this work
\end{abstract}

Correspondence: Guankui Du

Department of Biochemistry and

Molecular Biology, Hainan Medical

University, Haikou, 57I I0I, People's

Republic of China

Tel +86-898-66893779

Fax +86-898-66893600

Email duguankui@I63.com

Jun Chu

Key Laboratory of Xin'an Medicine,

Ministry of Education, Anhui University of

Chinese Medicine, Hefei, 230038, Anhui,

People's Republic of China

Email chuj@ahtcm.edu.cn
Background: Betel nuts have long been used in traditional Chinese medicine. In our study, the bioactive components of betel nut were systematically investigated, and the main components and their target genes in the treatment of depression were predicted.

Methods: The metabolites of the kernels and peels were analyzed with a UPLC-MS/MS system. Mass spectrometry outcomes were annotated by MULTIAQUANT. "Compounddisease targets" were utilized to construct a pharmacology network.

Results: A total of 873 metabolites were identified, with a high abundance of flavonoids, alkaloids, and phenols. Moreover, the abundance of flavonoids, alkaloids, and phenols in the kernel was significantly higher than that in the peel. A high abundance of catechin, arginine, and phenylalanine was detected in the kernel, while a high abundance of arginine, arecoline, and aminobutyric acid was detected in the peel. Catechins and cyanoside were the most abundant flavonoids in the kernel and peel, respectively. Arecoline was the most abundant alkaloid. A total of 111 metabolites showed a significant difference between the kernels and peels. The relative abundance of 40 differential metabolites was higher than 100,000, including 14 primary metabolites, 12 flavonoids, 4 phenols, and 4 alkaloids. Among the 40 high abundance metabolites, 20 were higher in the kernel and 20 in the peel. In addition, the enrichment of metabolic pathways found that the kernel and peel of the fruit adopted different metabolic pathways for the synthesis of flavonoids and alkaloids. Network pharmacology prediction showed that 93 metabolites could target 141 depression-related genes. The main components of betel nut intervention in depression were predicted to include L-phenylalanine, protocatechuic acid, okanin, nicotinic acid, L-tyrosine, benzocaine, syringic acid, benzocaine, phloretic acid, cynaroside, and 3,4-dihydroxybenzaldehyde.

Conclusion: Betel nuts are rich in natural metabolites, and some of these metabolites can participate in the intervention of depression. In addition, the metabolites showed distinct characteristics between the kernel and peel. Therefore, it is necessary to comprehensively and rationally use betel nuts.

Keywords: betel nut, metabolites, flavonoid, alkaloids, phenols

\section{Introduction}

Betel nut (Betel catechu L.) is primarily produced in India, Malaysia, Taiwan, and Hainan of China. ${ }^{1}$ Betel nuts are widely used in traditional Chinese medicine (TCM). The Chinese Pharmacopoeia includes 51 ancient Chinese medicine prescriptions utilizing betel nut as a raw material. ${ }^{2}$ In TCM, betel nuts are utilized for deworming, bloating, abdominal pain, indigestion, malaria, and tenesmus. ${ }^{3}$ Modern medical research has shown that compounds in betel nuts can also intervene in 
diseases of the digestive system, nervous system, and cardiovascular system. ${ }^{3}$ Betel nuts have antiallergic effects, regulate blood sugar, and lower blood lipids. ${ }^{3}$

Researchers have reported that betel nuts are rich in natural active substances. $^{3-5}$ Fifty-nine compounds have been isolated and identified from betel nut. ${ }^{3}$ The natural components of betel nut mainly include alkaloids, fatty acids, tannins, and amino acids. $^{3}$ Arecoline is the main alkaloid, ${ }^{4}$ while the tannins mainly exist in the form of condensed tannins. ${ }^{5}$ Betel nut seeds contain approximately $14 \%$ fat with a higher content of linoleic acid. ${ }^{6}$ However, the low-abundance components in betel nuts may not have all been identified.

Several studies have shown that betel nut has antidepressant potential. $^{7-9}$ Betel nut extract can inhibit monoamine oxidase $\mathrm{A}$ and promote an increase in serotonin and norepinephrine. ${ }^{7,8}$ In addition, betel nut extract can effectively increase the content of norepinephrine (NE) and dopamine serotonin (5-HT) in mouse brain tissue. ${ }^{7,10}$ The crude extract of betel nut obtained from $70 \%$ methanol aqueous solution can effectively inhibit the activity of acetylcholinesterase. ${ }^{11}$ Betel nut extract increased the levels of the neurodevelopment-related proteins MBP, CNPase, and GSTpi. ${ }^{12}$ The antidepressant components of betel nut deserve further analysis.

Liquid chromatography-tandem mass spectrometry (LC-MS/MS) provides a vital tool to analyze TCM metabolites systematically. Systematic evaluation of the betel nut composition has not been carried out; thus, the medicinal value of betel nut may be underestimated. In the present study, the peel and kernel of betel nut were collected, and untargeted metabolomics analysis was carried out by LC-MS/MS. Furthermore, network pharmacology analysis would help us comprehensively understand the medicinal value of betel nut, especially its antidepressant potential.

\section{Materials and Methods Plant Materials}

In June 2020, fresh betel nut samples were harvested from 6-year-old betel nut trees in the betel nut planting area of Yongxing Town, Haikou, China, and identified by Professor Jin Dejun. The collected betel nuts were in the mature stage and green, and the fruit weight varied from $25.4 \mathrm{~g}$ to $27.1 \mathrm{~g}$. The voucher specimen was deposited at the School of Pharmacy, Hainan Medical University.

\section{Metabolite Extraction}

After the fresh betel nut samples were freeze-dried, they were ground into a powder with a grinder. Then, $100 \mathrm{mg}$ of powder was dissolved in $1 \mathrm{~mL}$ of $70 \%$ methanol-water solution and refrigerated overnight at $4{ }^{\circ} \mathrm{C}$. The extract was centrifuged $\left(4^{\circ} \mathrm{C}, 10,000 \mathrm{~g}\right.$, and $10 \mathrm{~min}$ ) to obtain the supernatant. The supernatant was filtered through a microporous membrane filter $(0.22 \mu \mathrm{m}$ pore size). The sample was stored until subsequent analysis.

\section{Untargeted Metabolomic Analysis}

An ultrahigh-performance liquid chromatography (UPLC, Shim-pack UFLC SHIMADZU CBM30A)-tandem mass spectrometry (MS/MS, Applied Biosystems 4500, QTRAP) system was used for sample analysis. First, a UPLC system, a Waters ACQUITY UPLC HSS T3 C18 column $(2.1 \mathrm{~mm} \times 100 \mathrm{~mm}, 1.8 \mu \mathrm{m}$ particle size $)$ was used to separate the components (Waters, Herts, UK). The column temperature was $40{ }^{\circ} \mathrm{C}$, and the flow rate was $0.4 \mathrm{~mL} / \mathrm{min}$. The mobile phase consisted of solvent A (water containing $0.04 \%$ acetic acid) and solvent $\mathrm{B}$ (acetonitrile containing $0.04 \%$ acetic acid). The gradient elution conditions were as follows: $0 \mathrm{~min}, 95: 5 \mathrm{~V} / \mathrm{V}(\mathrm{A} / \mathrm{b})$; $0 \mathrm{~min}, 5: 95 \mathrm{~V} / \mathrm{V} ; 0 \mathrm{~min}, 5: 95 \mathrm{~V} / \mathrm{V} ; 1 \mathrm{~min}, 95: 5 \mathrm{~V} / \mathrm{V}$; and 15.0 minutes, $95: 5 \mathrm{~V} / \mathrm{v}$.

High-resolution MS/MS was used to detect the components separated by UPLC. The temperature of the electrospray ionization was 550 degrees. The MS voltage was 5500 v. The curtain gas was 25 psi. The collision activation dissociation was set to high. After the component analysis, the mass spectrum peaks needed to be corrected. The multiple reaction monitoring (MRM) mode of the triple quadrupole mass spectrometry was used for quantification of the metabolites. ${ }^{13}$ Mixed samples were used for the quality control and repeatability of the testing instruments.

\section{Bioinformatics of the Untargeted Metabolism (for TCM) Dataset}

The original UPLC-MS/MS data needed to be analyzed and identified by various software programs and databases. Analyst 1.6.3 software was used to generate the molecular characteristic matrices, such as the retention time and mass charge ratio $(\mathrm{M} / \mathrm{Z})$. The following databases were used for the structural analysis of the metabolites: MASSBANK (http://www.massbank.jp/), KNAPSAcK (http://kanaya. naist.jp/KNApSAcK/), HMDB (http://www.hmdb.ca/), ${ }^{14}$ 
MoTo DB (http://www.ab.wur.nl/moto/) and METLIN (http://metlin.scripps.edu/index.php). ${ }^{15}$

After obtaining the mass spectrum data of the sample metabolites, the peaks of the same metabolites in different samples were calculated and corrected. ${ }^{13}$

MULTIAQUANT software was used to open the mass spectrum file of each sample and integrate the peaks. The peak area of the chromatographic peak represented the relative content of the corresponding substance.

\section{Target Identification and Network Construction}

The compound targets were searched for in the SWISSADME (http://www.swissadme.ch/) ${ }^{16}$ and TargetNet (http://targetnet. scbdd.com/calcnet/index/) databases, ${ }^{17}$ which use various algorithms to predict the potential target compounds. Briefly, the SwissTargetPrediction plug-in in the SWISSADME website was used for the target gene analysis. We selected a species and then pasted a SMILES, which could be found in the PubChem database (https://pubchem.ncbi.nlm.nih.gov/), into the box. The TargetNet website performed a similar operation. After entering SMILES into the dialog box, the default option was adopted (AUC $\geq 0.7$, fingerprint type: ECFP4). More than $95 \%$ of the probability targets were included among the disease-associated targets.

To compile the disease targets for depression, the GeneCards database was searched. ${ }^{18}$ The intersection between the drug and disease targets was used to screen the key targets. Cytoscape software was used to build the network. ${ }^{19}$

\section{Systematic Correlativity Analysis and Statistical Analysis}

Pearson's correlation, one-way analysis of variance (ANOVA), and hierarchical (average linkage) clustering were performed for the untargeted metabolic analyses. $P$ values of the ANOVA were adjusted for the false discovery rate.

\section{Results}

Betel nuts can be divided into kernels and peels (Figure 1A). A total of 873 metabolites were identified by UPLC-MS/ MS analysis (Figure S1 and Table S1), including 174 primary metabolites and 699 secondary metabolites. Multiple primary metabolites were determined, including 50 amino acids and their derivatives, 37 lipid compounds, 41 nucleotides and their derivatives, and 13 carbohydrates. Meanwhile, 699 secondary metabolites could be classified into flavonoids (143), terpenes (104), alkaloids (102), phenols (79), lignans, coumarins (41), phenylpropanoids (33), quinones (26), and others (81) (Figure 1B).

The total abundance of metabolites of the kernel was $643,629,320$, while that of the peel was $198,919,279$. Primary metabolites accounted for $40.5 \%$ in the kernel and $54.4 \%$ in the peel (Figure 1C). The top 3 most abundant secondary metabolites were flavonoids, alkaloids, and phenols in the kernel. Alkaloids, flavonoids, and phenols were the top 3 metabolites in the peel. Flavonoids accounted for $30.1 \%$ and $6 \%$ in the kernel and peel, respectively. Phenolics accounted for approximately $5.5 \%$ of both kernels and peels. Alkaloids accounted for $9.7 \%$ and $16.8 \%$ of the kernel and peel, respectively. Moreover, the abundance of flavonoids, alkaloids, and phenols in the kernel was 15.5-fold, 3.2-fold, and 1.8-fold that in the peel, respectively.

The top 10 most abundant metabolites of the kernel were cianidanol, L-arginine, L-phenylalanine, benzocaine, arecoline, epicatechin, sucrose, turanose, lactulose, and 3,4-dihydroxybenzaldehyde (Table 1). Meanwhile, the top 10 most abundant metabolites of the peel were L-arginine, arecoline, D-alpha-aminobutyric acid, sucrose, D-maltose, turanose, lactulose, L-citrulline, trigonelline, and p-octopamine.

As shown in Figure 2A, a total of 111 differential metabolites were found (fold change $>2, \mathrm{P}<0.05$ ) (Figure 2A). Among them, 40 metabolites (abundance over 100,000 in the kernel and/or peel) had a high abundance, including 14 primary metabolites and 26 secondary metabolites (Figure 2B). Cluster analysis showed that 20 metabolites had a higher accumulation in the kernel, while the other 20 metabolites accumulated more in the peel. Among the 14 primary metabolites, 8 were abundant in the peel. Seven flavonoids each had high abundances in the kernel and peel. Two of the 4 phenols had a higher abundance in the kernel.

Various amino acids with a high abundance were isolated by LC-MS/MS; thus, the amino acids and their derivatives were analyzed. Twenty-three amino acids and their derivatives with a high abundance were found (Figure 3A). Proline, L-citrulline, L-lysine, L-glutamic acid, and L-pipecolic acid were highly accumulated in both the kernels and peels. The abundance of tyrosine, phenylalanine, arginine, serine, and histidine in the kernel was significantly higher than that in the peel. Meanwhile, 4-aminobutyric acid in the kernel was significantly lower than that in the peel. Furthermore, the analysis of the amino acid synthesis pathways showed that the synthesis of multiple amino acids was more active in the kernel than in the peel (Figure 3B). 
A

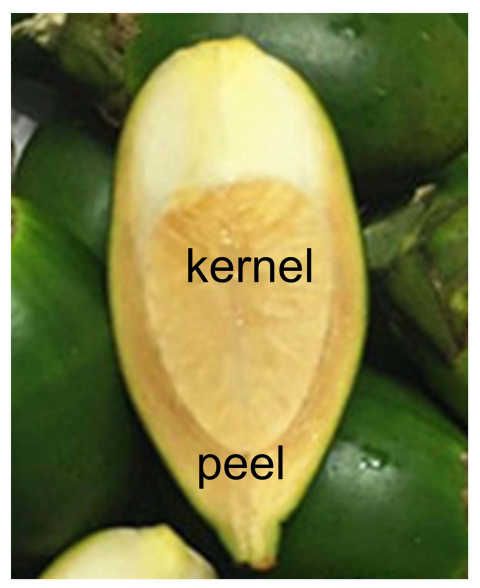

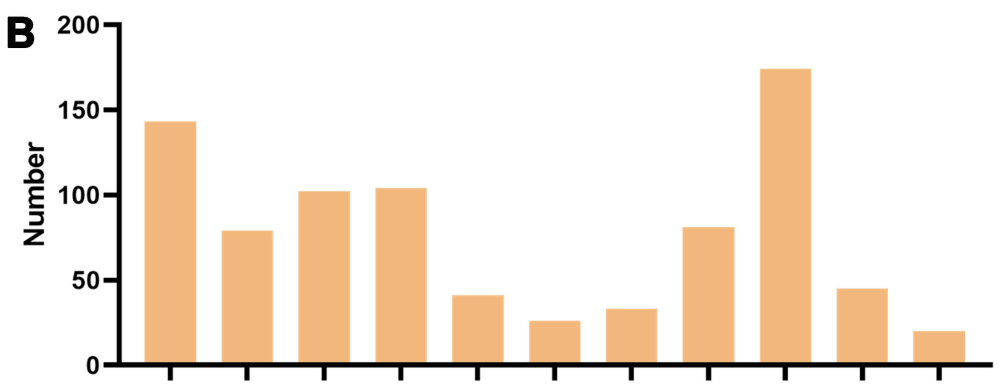

C

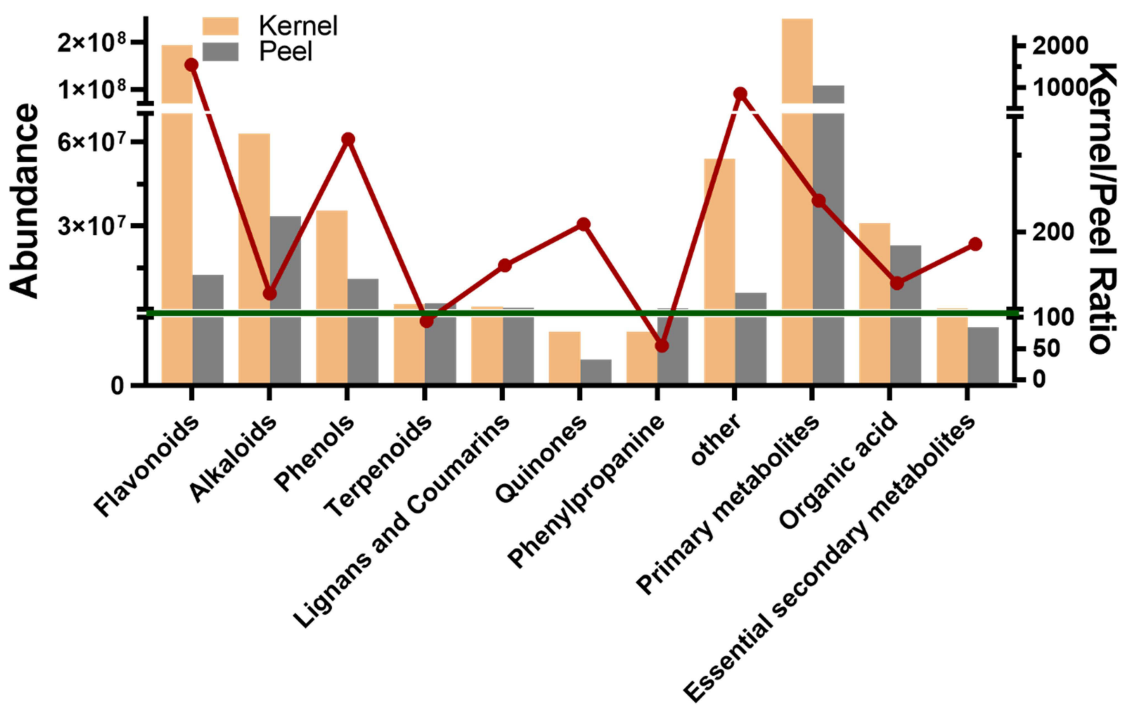

Figure I Untargeted metabolite profiling identified the metabolites in the betel nut. (A) The position of the betel nut kernel and peel. (B) The differentially accumulated metabolites were assigned to various secondary metabolic categories. (C) Abundance of metabolites and the ratio of metabolite abundance in kernels/peels.

The abundance of 26 flavonoids was found to be higher than 100,000 in the kernel and/or peel (Figure 4A). The abundances of rutin, cianidanol, procyanidin b1, narcissoside, okanin, procyanidin b2, L-epicatechin epicatechin, vestitol, and kaempferol-3-o-rutinoside in the kernel were more than 10 -fold higher than those in the peel. In contrast, tectorigenin, homoorientin, cynaroside, peonidin-3-glucoside, saponarin, vitexin-2-glucoside, meloside a, and pelargonidin-3,5-o-diglucoside chloride in the peel were more than 10-fold higher than those in the kernel. In addition, the enrichment of the metabolic pathways showed that the synthesis of the flavonoids was more active in the kernel than in the peel (Figure 4B).

Table I The Top Ten Metabolites in Betel Nut Kernel and Peel

\begin{tabular}{|l|r|l|r|}
\hline \multicolumn{2}{|c|}{ Kernel } & \multicolumn{2}{c|}{ Peel } \\
\hline \multicolumn{1}{|c|}{ Metabolites } & Abundance & \multicolumn{1}{|c|}{ Metabolites } & Abundance \\
\hline Cianidanol & $152,471,359$ & L-Arginine & $28,793,352$ \\
L-Arginine & $119,344,455$ & Arecoline & $12,864,899$ \\
L-Phenylalanine & $45,625,317$ & D-alpha-Aminobutyric acid & $10,304,963$ \\
Benzocaine & $45,625,317$ & Sucrose & $9,007,384$ \\
Arecoline & $38,188,414$ & D-Maltose & $8,775,721$ \\
Epicatechin & $12,526,820$ & Turanose & $8,380,532$ \\
Sucrose & $10,811,009$ & Lactulose & $8,182,635$ \\
Turanose & $10,671,334$ & L-Citruline & $8,077,661$ \\
Lactulose & $10,483,274$ & Trigonelline & $7,592,720$ \\
3,4-Dihydroxybenzaldehyde & $10,086,418$ & P-Octopamine & $6,797,788$ \\
\hline
\end{tabular}



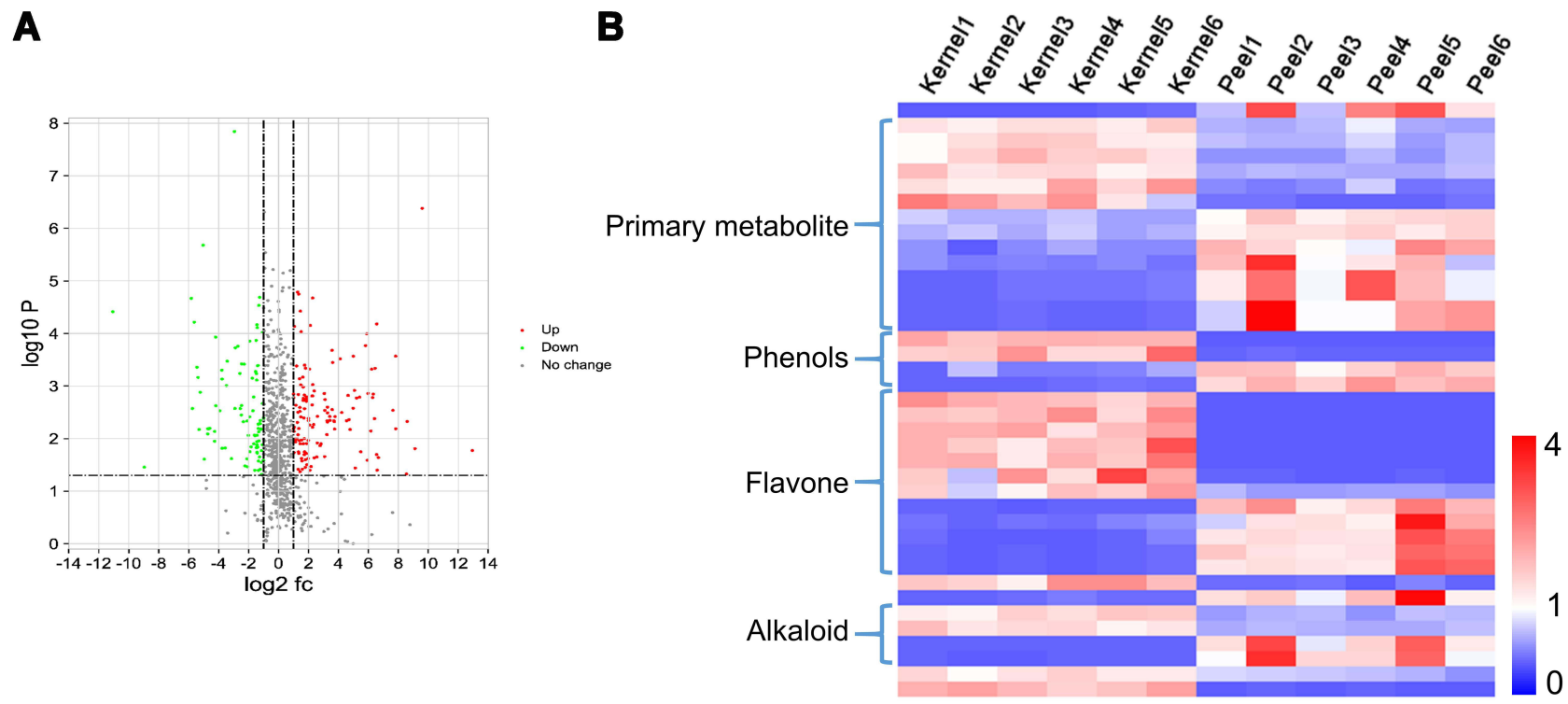

Figure 2 Screening of differential metabolites. (A) A total of I I I differentially expressed metabolites were screened by volcanic mapping $(P<0.05$, fold change $>2)$. (B) A heatmap of the relative amounts of differentially accumulated metabolites, whose relative abundance was more than 100,000. Of the 40 metabolites, 14 were primary metabolites. Among the 26 secondary metabolites, there were 4 phenols, 12 flavonoids, and 4 alkaloid metabolites. The abundance of 20 metabolites was higher in the kernel and that of 20 metabolites was higher in the peel. The heatmap scale ranges from -1 to +4 after data homogenization (ratio of the measured value to the average value).

Twelve alkaloid metabolites with high abundance were isolated (Figure 5A). Among them, the phyllalbine content in the kernel was 75 -fold higher than that in the peel.
Cinchonidine and cinchonine in the peel were 32- and 41fold higher, respectively, than those in the kernel. Notably, a high abundance of arecoline was detected in both the
A

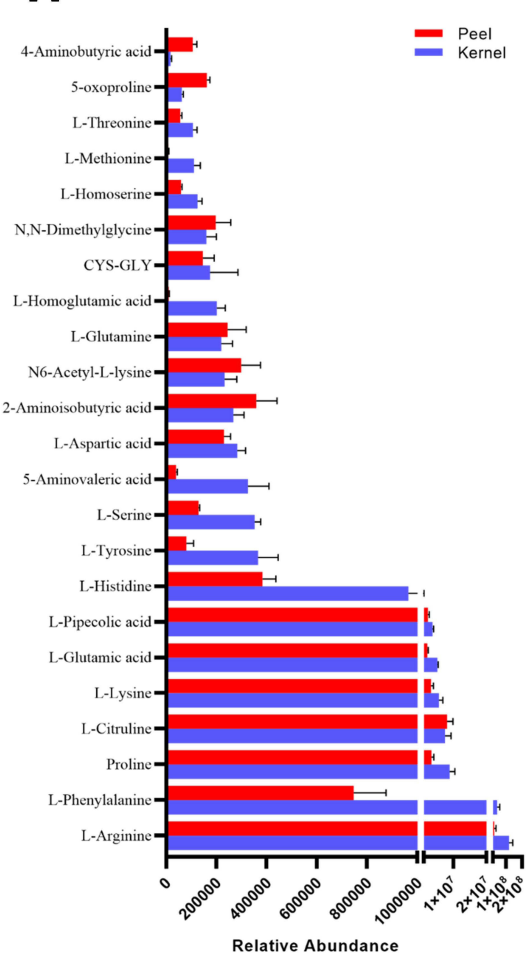

B

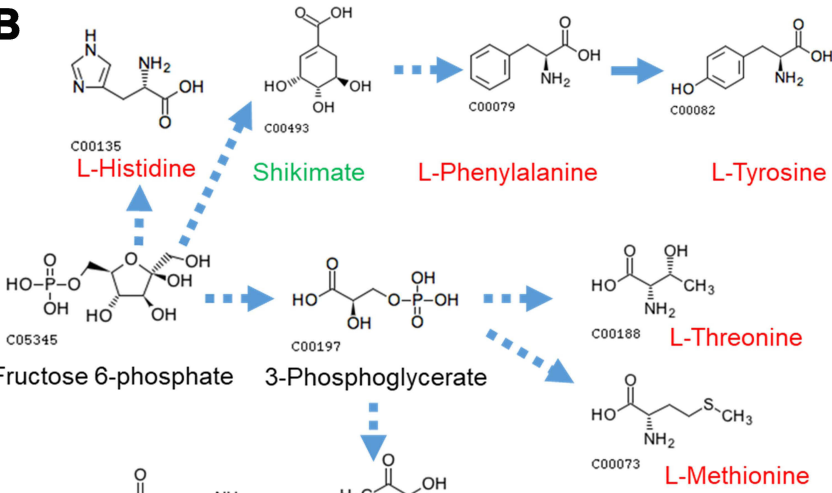

Figure 3 Accumulation of amino acids and their derivatives in the kernel and peel. (A) Relative abundance of amino acids and their derivatives in kernels and peels. (B) Enrichment analysis of the amino acid biosynthesis pathway. Starting from fructose 6 phosphate, there were significant differences in the biosynthesis of various amino acids in betel nuts. Red indicates higher abundance in the kernel, while green indicates higher abundance in the peel. 


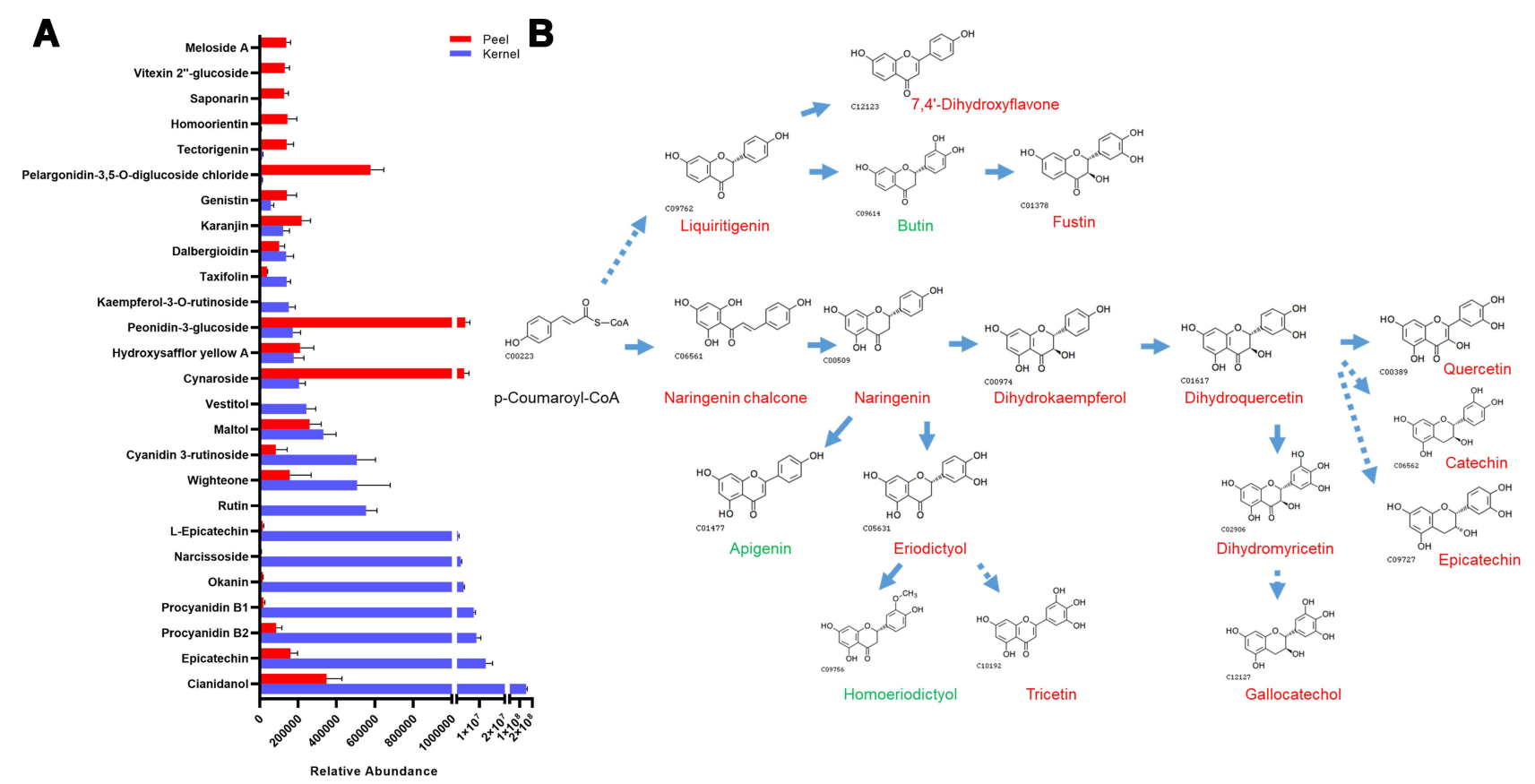

Figure 4 Accumulation of flavonoids in the kernel and peel. (A) Relative abundance of flavonoids in the kernel and peel. (B) Enrichment analysis of the flavonoid biosynthesis pathway. Starting from p-Coumaroyl-CoA, flavonoids were synthesized through two metabolic pathways, Liquiritigenin and Naringenin chalcone. Red indicates a higher abundance in the kernel, while green indicates a higher abundance in the peel.

kernel and peel. The abundance of arecoline in the kernel was 2.9-fold that in the peel.

Sixteen phenolic metabolites had a high abundance in betel nut (Figure 5B). The abundance of 3,4-dihydr oxybenzaldehyde, trimethoprim, phloretic acid, and 2,5-dihydroxybenzaldehyde in the kernel was much higher than that in the peel. In addition, the abundance of dihydromethysticin, vanillic acid, and 6-gingerol in the peel was much higher than that in the kernel.

Based on the metabolites obtained from the UPLC-MS/ MS analysis, the components of betel nut that might be useful in the treatment of depression were predicted by network pharmacology (Figure 6). A total of 93 high abundance metabolites were found to target 141 genes. The results showed that L-phenylalanine, protocatechuic acid, okanin, nicotinic acid, L-tyrosine, benzocaine, syringic acid, benzocaine, phloretic acid, cynaroside, and 3,4-dihydroxybenzaldehyde could play a major role in the intervention of depression. In addition, these metabolites mainly interacted with genes such as CES2, ARG1, NOS2, GCLC, NOS3, RIA1, VEGFA, CYP1B1, TH, ODC1, and GSTM1.

\section{Discussion}

Chewing betel nut is popular all over the world. ${ }^{20}$ Hundreds of millions of people worldwide chew betel nuts. ${ }^{20}$ Chewing betel nut is primarily popular in the
Asia Pacific region. ${ }^{1}$ Our previous research showed that the chewing rate of betel nuts in rural areas of Hainan was $43.84 \%$ for men and $7.43 \%$ for women (data not shown). The main reason for the popularity of betel nut is because it is addictive, similar to the use of tobacco. ${ }^{20}$ Chewing betel nut can induce oral cancer through various mechanisms, such as promoting the fibrosis of oral epithelial cells, enhancing the drug resistance of cancer cells, and changing the oral microecology. ${ }^{21-23}$ However, the effects of betel nut on human health may be two-sided. Studies have shown that betel nut can resist migraines, reduce cholesterol levels, and regulate glucose metabolism. ${ }^{24-26}$ In this study, the metabolites of betel nut were systematically analyzed to provide a basis for the rational utilization of betel nut.

In this study, 873 metabolites were found and categorized into 11 classes. Flavonoids, alkaloids, and phenols were the most abundant secondary metabolites. Previous studies suggested that there were 59 main metabolites of betel nut, mainly alkaloids and flavonoids. ${ }^{3}$ The present study not only isolated most of the reported metabolites, such as catechins, epicatechins, and isorhamnetin but also highlighted hundreds of unique, never previously reported metabolites from betel nut.

This study clarified the metabolite differences between kernels and peels. A total of 111 metabolites with significant 


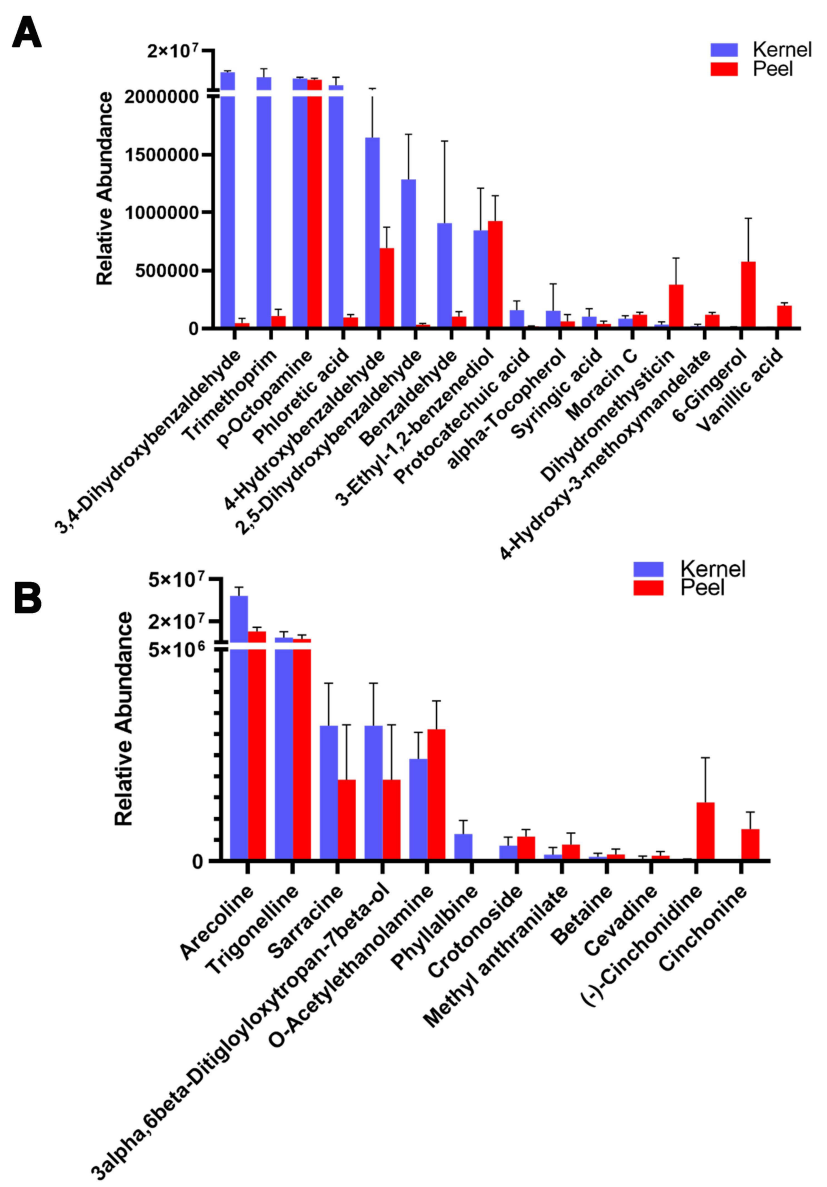

Figure 5 Accumulation of alkaloids and phenols in the kernel and peel. (A) Relative abundance of phenols in the kernel and peel. Various high-abundance phenolic metabolites were higher in the kernel than in the peel. (B) Relative abundance of alkaloids in the kernel and peel. The abundances of arecoline and trigonelline were high in both kernels and peels.

differences were identified. Previous studies have mainly focused on the metabolite components of the whole betel nut. ${ }^{27} \mathrm{~A}$ recent study analyzed the distribution of arecoline in distinct regions of the nut. ${ }^{28}$ This study first systematically elucidated the distribution of metabolites and the differences in metabolites between kernels and peels.

This study revealed that flavonoids were the most abundant metabolites in both the kernel and peel. Catechin had the highest abundance of metabolites in the betel nut. Several flavonoids have been identified in previous studies, including isorhamnetin, quercetin, liquiritigenin, catechin, proanthocyanidins, and epicatechin. ${ }^{29-32}$ Catechins have multiple functions. It has been noted that catechins powerfully decrease the endogenous formation of nitrosation. ${ }^{32}$ Catechins possess anti-inflammatory and antiplatelet activity and reduce cholinomimetic activity potential in vivo. ${ }^{33-35} \mathrm{In}$ addition, recent studies showed that betel nut procyanidins could improve hyperglycemia by regulating gluconeogenesis 26 and inducing lymphocyte apoptosis. ${ }^{36}$ Therefore, the flavonoids in betel nut may be the main source of its biological activity.

A total of 102 alkaloids were obtained by UPLC-MS/ MS analysis, among which arecoline was the most abundant. Previous studies have shown that arecoline is the main alkaloid in betel nut. ${ }^{37,38}$ In addition, a variety of alkaloids have been identified. ${ }^{27,37}$ Moreover, arecoline has a variety of physiological effects and is considered to be a representative substance of betel nuts. ${ }^{39}$ This study confirmed that arecoline is the dominant alkaloid in betel nuts.

In addition, this study highlighted that phenols were the dominant metabolites in betel nuts. A total of 79 phenolic substances were found in betel nut, and their abundance was the third dominant metabolite. In previous studies, a variety of phenolic substances were extracted from betel nut, such as ferulic acid, vanillic acid, caffeic acid and ferulic acids. ${ }^{29,40}$ The phenolic compounds in betel nut have multiple functions, such as antiaging and antiplaque activities. ${ }^{41,42}$ However, previous studies have shown that polyphenols might induce tumors ${ }^{43}$ and boost the development of myeloid-derived suppressor cells. ${ }^{44}$ Therefore, the effects of phenolic metabolites derived from betel nut needs further research.

Network pharmacology predicted the main antidepressant components in betel nut. Amino acids and their derivatives (such as phenylalanine and tyrosine) and phenols (including protocatechuic acid, syringic acid, phloretic acid, and 3,4-dihydroxybenzaldehyde) may be the main bioactive components. Studies have shown that the levels of tyrosine and phenylalanine in the circulatory system of patients with depression are reduced. $^{45}$ Tyrosine is considered to be the starting material for the synthesis of dopamine. A decrease in dopamine is directly related to depression. ${ }^{45}$ Arecoline and total phenolic compounds of betel nut could significantly shorten the immobility time of mice, and total phenolic compounds showed better antidepressant effects. ${ }^{3}$ The alkaloids and phenols in betel catechu can effectively bind to the MAO-A receptor, but phenols more easily bind to the MAO-A receptor. ${ }^{8}$ Therefore, the main components of areca antidepressants include primary metabolites and secondary metabolites.

Meanwhile, network pharmacology predictions revealed that betel nut targets 141 genes (Table S2). KEGG functional enrichment suggested that these target genes are related to various inflammatory response 


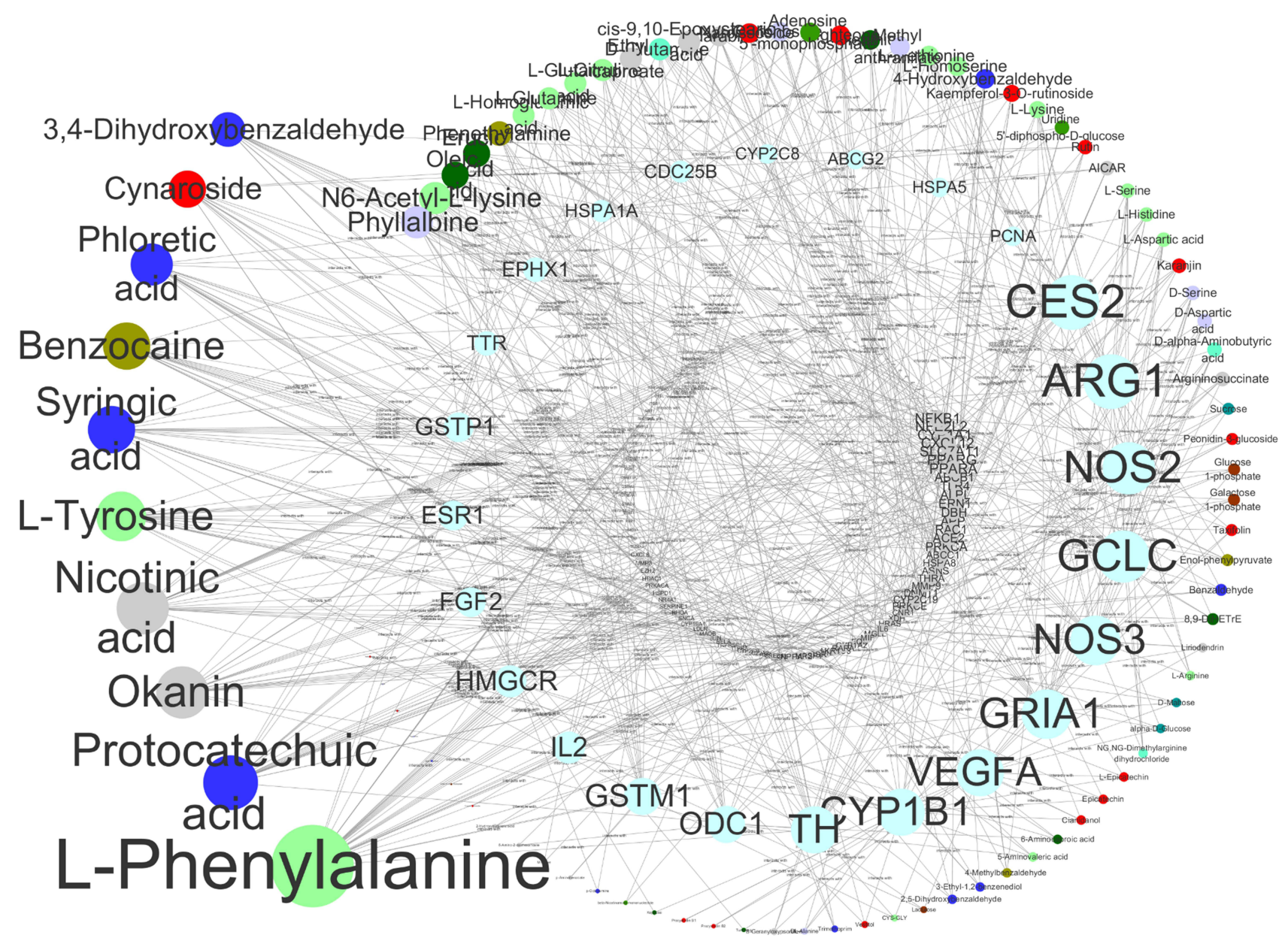

Figure 6 Network pharmacology analysis of major components and targets of antidepressants in betel nut. The main metabolites in betel nut that interfere with depression include L-phenylalanine, protocatechuic acid, okanin, nicotinic acid, L-tyrosine, benzocaine, syringic acid, benzocaine, phloretic acid, cynaroside, and 3,4-dihydroxybenzaldehyde. Moreover, the main genes targeted by betel nut metabolites included CES2, ARGI, NOS2, GCLC, NOS3, RIAI, VEGFA, CYPIBI, TH, ODCI, and GSTMI. The size of the circle was positively related to the number of connected edges.

pathways, including the IFN-gamma pathway, IL5mediated signaling events, IL3-mediated signaling events, CDC42 signaling events, Phase 1 Functionalization of compounds, TNF receptor signaling pathway, IL2-mediated signaling events, IL1-mediated signaling events, TGF-beta receptor signaling, IL23mediated signaling events, p38 MAPK signaling pathway, IL12-mediated signaling events, IL2 signaling events mediated by PI3K, PI3K/AKT activation, endogenous TLR signaling, and E-cadherin signaling in keratinocytes (Table S3). Depression is considered to be an inflammatory disease. ${ }^{46}$ These results suggest that betel nut may interfere with depression by acting on inflammatory signaling pathways.

\section{Conclusion}

In summary, flavonoids, alkaloids, and phenols were the main secondary metabolites in betel nut. Catechin and arecoline were representative active components of the betel nut. Network pharmacology predicted that betel nut might act on inflammation-related signaling pathways and be effective in the treatment of depression. The metabolites of the kernel and peel were significantly different; thus, the effective and rational utilization of betel nut might be worthy of further study.

\section{Data Sharing Statement}

All datasets generated and analyzed during the current study were uploaded with the manuscript as additional files.

\section{Ethics Statement}

None required.

\section{Author Contributions}

All authors made a significant contribution to the work reported, whether that is in the conception, study 
design, execution, acquisition of data, analysis and interpretation, or in all these areas; took part in drafting, revising or critically reviewing the article; gave final approval of the version to be published; have agreed on the journal to which the article has been submitted; and agree to be accountable for all aspects of the work.

\section{Funding}

This study was funded by the Natural Science Foundation of Hainan (821RC561), and Innovative research projects for graduate students in Hainan Province (Hys2020-350).

\section{Disclosure}

The authors report no conflicts of interest in this work.

\section{References}

1. Mehrtash H, Duncan K, Parascandola M, et al. Defining a global research and policy agenda for betel quid and areca nut. Lancet Oncol. 2017;18(12):e767-e775. doi:10.1016/S1470-2045(17)30460-6

2. Sun L, Song HB, Zhang L, et al. Systematic evaluation for safety of traditional Chinese medicine Areca catechu and its preparations. Zhongguo Zhong Yao Za Zhi. 2017;42(21):4067-4073. doi:10.19540/j.cnki.cjemm.20170919.014

3. Peng W, Liu YJ, Wu N, et al. Areca catechu L. (Arecaceae): a review of its traditional uses, botany, phytochemistry, pharmacology and toxicology. J Ethnopharmacol. 2015;164:340-356. doi:10.1016/j.jep.2015.02.010

4. Wu J, Zhang H, Wang S, et al. Tissue-specific and maturity-dependent distribution of pyridine alkaloids in Areca triandra. $J$ Plant Res. 2019;132(4):531-540. doi:10.1007/s10265-019-01115-9

5. Noor Awang M. Quantitative analysis of areca catechu (betel) nut flavanols (tannins) in relation to oral submucous fibrosis. Dent J Malays. 1987;9(2):29-32.

6. Pithayanukul P, Nithitanakool S, Bavovada R. Hepatoprotective potential of extracts from seeds of Areca catechu and nutgalls of Quercus infectoria. Molecules. 2009;14(12):4987-5000. doi:10.3390/ molecules 14124987

7. Abbas G, Naqvi S, Erum S, et al. Potential antidepressant activity of Areca catechu nut via elevation of serotonin and noradrenaline in the hippocampus of rats. Phytother Res. 2013;27(1):39-45. doi:10.1002/ ptr.4674

8. Dar A, Khatoon S. Behavioral and biochemical studies of dichloromethane fraction from the Areca catechu nut. Pharmacol Biochem Behav. 2000;65(1):1-6. doi:10.1016/S0091-3057(99)00179-3

9. Dar A, Khatoon S, Rahman G, et al. Anti-depressant activities of Areca catechu fruit extract. Phytomedicine. 1997;4(1):41-45. doi:10.1016/S0944-7113(97)80026-8

10. Inokuchi J, Okabe H, Yamauchi T, et al. Antihypertensive substance in seeds of Areca catechu L. Life Sci. 1986;38(15):1375-1382. doi:10.1016/0024-3205(86)90470-4

11. Gilani AH, Ghayur MN, Saify ZS, et al. Presence of cholinomimetic and acetylcholinesterase inhibitory constituents in betel nut. Life Sci. 2004;75(20):2377-2389. doi:10.1016/j.lfs.2004.03.035

12. Adilijiang A, Guan T, Xu ZZ, et al. The aqueous fraction of areca catechu nut ameliorates demyelination in prefrontal cortex-induced depressive symptoms and cognitive decline through brain-derived neurotrophic factor-cyclic adenosine monophosphate response element-binding activation. Chin J Integr Med. 2016. doi:10.1007/ s11655-016-2455-8
13. Fraga C, Clowers B, Moore R, et al. Signature-discovery approach for sample matching of a nerve-agent precursor using liquid chromatography-mass spectrometry, XCMS, and chemometrics. Anal Chem. 2010;82(10):4165-4173.

14. Wishart D, Jewison T, Guo A, et al. HMDB 3.0-the human metabolome database in 2013. Nucleic Acids Res. 2013;41:D801-807. doi:10.1093/nar/gks1065

15. Montenegro-Burke J, Guijas C, Siuzdak G. METLIN: a tandem mass spectral library of standards. Methods Mol Biol. 2020;2104:149-163.

16. Daina A, Michielin O, Zoete V. SwissADME: a free web tool to evaluate pharmacokinetics, drug-likeness and medicinal chemistry friendliness of small molecules. Sci Rep. 2017;7:42717. doi:10.1038/srep42717

17. Yao Z, Dong J, Che Y, et al. TargetNet: a web service for predicting potential drug-target interaction profiling via multi-target SAR models. J Comput Aided Mol Des. 2016;30(5):413-424. doi:10.1007/s10822-016-9915-2

18. Stelzer G, Rosen N, Plaschkes I, et al. The genecards suite: from gene data mining to disease genome sequence analyses. Curr Protoc Bioinformatics. 2016;54:1-30.

19. Shannon P, Markiel A, Ozier O, et al. Cytoscape: a software environment for integrated models of biomolecular interaction networks. Genome Res. 2003;13(11):2498-2504. doi:10.1101/gr.1239303

20. Athukorala IA, Tilakaratne WM, Jayasinghe RD. Areca nut chewing: initiation, addiction, and harmful effects emphasizing the barriers and importance of cessation. J Addict. 2021;2021:9967097. doi:10.1155/ 2021/9967097

21. Wang L, Tang Z. Immunopathogenesis of oral submucous fibrosis by chewing the areca nut. $J$ Leukoc Biol. 2021. doi:10.1002/ JLB.3MR0521-763RR

22. Li YC, Cheng AJ, Lee LY, et al. Multifaceted mechanisms of areca nuts in oral carcinogenesis: the molecular pathology from precancerous condition to malignant transformation. $J$ Cancer. 2019;10 (17):4054-4062. doi:10.7150/jca.29765

23. Hernandez BY, Zhu X, Goodman MT, et al. Betel nut chewing, oral premalignant lesions, and the oral microbiome. PLoS One. 2017;12 (2):e0172196. doi:10.1371/journal.pone.0172196

24. Bhandare AM, Vyawahare NS, Kshirsagar AD. Anti-migraine effect of Areca Catechu L. nut extract in bradykinin-induced plasma protein extravasation and vocalization in rats. $J$ Ethnopharmacol. 2015;171:121-124. doi:10.1016/j.jep.2015.05.052

25. Huang PL, Chi CW, Liu TY. Areca nut procyanidins ameliorate streptozocin-induced hyperglycemia by regulating gluconeogenesis. Food Chem Toxicol. 2013;55:137-143. doi:10.1016/j.fct.2012.12.057

26. Park YB, Jeon SM, Byun SJ, et al. Absorption of intestinal free cholesterol is lowered by supplementation of Areca catechu L. extract in rats. Life Sci. 2002;70(16):1849-1859. doi:10.1016/ S0024-3205(02)01485-6

27. Cao M, Yuan H, Daniyal M, et al. Two new alkaloids isolated from traditional Chinese medicine Binglang the fruit of Areca catechu. Fitoterapia. 2019;138:104276. doi:10.1016/j.fitote.2019.104276

28. Srimany A, George C, Naik HR, et al. Developmental patterning and segregation of alkaloids in areca nut (seed of Areca catechu) revealed by magnetic resonance and mass spectrometry imaging. Phytochemistry. 2016;125:35-42. doi:10.1016/j.phytochem.2016.02.002

29. Yang WQ, Wang HC, Wang WJ, et al. Chemical constituents from the fruits of Areca catechu. Zhong Yao Cai. 2012;35 (3):400-403.

30. Wu Q, Yang Y, Simon JE. Qualitative and quantitative HPLC/ MS determination of proanthocyanidins in areca nut (Areca catechu). Chem Biodivers. 2007;4(12):2817-2826. doi:10.1002/ cbdv.200790231

31. Pawar AB, More SP, Adivarekar RV. Dyeing of polyester and nylon with semi-synthetic azo dye by chemical modification of natural source areca nut. Nat Prod Bioprospect. 2018;8(1):23-29. doi:10.1007/s13659-017-0144-8 
32. Stich HF, Ohshima H, Pignatelli B, et al. Inhibitory effect of betel nut extracts on endogenous nitrosation in humans. J Natl Cancer Inst. 1983;70(6):1047-1050.

33. Huang PL, Chi CW, Liu TY. Effects of Areca catechu L. containing procyanidins on cyclooxygenase-2 expression in vitro and in vivo. Food Chem Toxicol. 2010;48(1):306-313. doi:10.1016/j.fct.2009.10.014

34. Ghayur MN, Kazim SF, Rasheed H, et al. Identification of antiplatelet and acetylcholinesterase inhibitory constituents in betel nut. Zhong Xi Yi Jie He Xue Bao. 2011;9(6):619-625. doi:10.3736/jcim20110607

35. Ghayur MN, Gilani AH. Cardio-selective inhibitory effect of the betel nut extract: possible explanation. Pharmazie. 2007;62(1):67-71.

36. Wang CC, Huang PL, Liu TY, et al. Highly oligomeric procyanidins from areca nut induce lymphocyte apoptosis via the depletion of intracellular thiols. Toxicol in Vitro. 2009;23(7):1234-1241. doi:10.1016/j.tiv.2009.07.033

37. Tang SN, Zhang J, Liu D, et al. Three new areca alkaloids from the nuts of Areca catechu. J Asian Nat Prod Res. 2017;19 (12):1155-1159.

38. Peng W, Liu YJ, Hu MB, et al. Using the "target constituent removal combined with bioactivity assay" strategy to investigate the optimum arecoline content in charred areca nut. Sci Rep. 2017;7:40278. doi:10.1038/srep40278

39. Liu YJ, Peng W, Hu MB, et al. The pharmacology, toxicology and potential applications of arecoline: a review. Pharm Biol. 2016;54 (11):2753-2760. doi:10.3109/13880209.2016.1160251

40. Stich HF, Dunn BP, Pignatelli B, et al. Dietary phenolics and betel nut extracts as modifiers of $\mathrm{N}$-nitrosation in rat and man. IARC Sci Publ. 1984;57:213-222.
41. Iwamoto M, Uchino K, Toukairin T, et al. The growth inhibition of Streptococcus mutans by 5'-nucleotidase inhibitors from Areca catechu L. Chem Pharm Bull (Tokyo). 1991;39(5):1323-1324. doi:10.1248/cpb.39.1323

42. Lee KK, Cho JJ, Park EJ, et al. Anti-elastase and anti-hyaluronidase of phenolic substance from Areca catechu as a new anti-ageing agent. Int $J$ Cosmet Sci. 2001;23(6):341-346. doi:10.1046/j.04125463.2001.00102.x

43. Wang D, Zhou L, Li W, et al. Anticoccidial effects of areca nut (Areca catechu L.) extract on broiler chicks experimentally infected with Eimeria tenella. Exp Parasitol. 2018;184:16-21. doi:10.1016/j. exppara.2017.11.002

44. Wang CC, Lin HL, Liang HJ, et al. Areca nut extracts enhance the development of $\mathrm{CD} 11 \mathrm{~b}(+) \mathrm{Gr}-1(+)$ cells with the characteristics of myeloid-derived suppressor cells in antigen-stimulated mice. J Oral Pathol Med. 2011;40(10):769-777. doi:10.1111/j.1600-0714.20 11.01043.x

45. Bekhbat M, Treadway MT, Goldsmith DR, et al. Gene signatures in peripheral blood immune cells related to insulin resistance and low tyrosine metabolism define a sub-type of depression with high CRP and anhedonia. Brain Behav Immun. 2020;88:161-165. doi:10.1016/ j.bbi.2020.03.015

46. Mac Giollabhui N, Ng TH, Ellman LM, et al. The longitudinal associations of inflammatory biomarkers and depression revisited: systematic review, meta-analysis, and meta-regression. Mol Psychiatry. 2020;26:1-3.

\section{Publish your work in this journal}

Drug Design, Development and Therapy is an international, peerreviewed open-access journal that spans the spectrum of drug design and development through to clinical applications. Clinical outcomes, patient safety, and programs for the development and effective, safe, and sustained use of medicines are a feature of the journal, which has also been accepted for indexing on PubMed Central. The manuscript management system is completely online and includes a very quick and fair peer-review system, which is all easy to use. Visit http://www. dovepress.com/testimonials.php to read real quotes from published authors. 